\title{
Dust Extinction toward SN 2014J in M82
}

\author{
Jian Gao ${ }^{1}$, B. W. Jiang ${ }^{1,2}$, and Aigen $\mathbf{L i}^{2}$ \\ ${ }^{1}$ Department of Astronomy, Beijing Normal University, \\ Beijing 100875, China \\ email: jiangao@bnu.edu.cn, bjiang@bnu.edu.cn \\ ${ }^{2}$ Department of Physics and Astronomy, University of Missouri, \\ Columbia, MO 65211, USA \\ email: lia@missouri.edu
}

\begin{abstract}
We derive the extinction toward SN 2014J, a Type Ia supernova in M82, as a function of wavelength from the far ultraviolet to the near infrared by modeling the observed color excesses in terms of a mixture of silicate and graphite. With $A_{V} \approx 1.9$ mag and $R_{V} \approx 1.7-1.8$, the derived extinction law differs substantially from those of the Galaxy and the Magellanic clouds.
\end{abstract}

Keywords. dust, extinction - galaxies: ISM - supernovae: individual (SN 2014J)

SN 2014J, in the nearby starburst galaxy M82, is the nearest type Ia supernova (SNe Ia) discovered in the last three decades. Its proximity offers an unprecedented opportunity to study the extinction and reddening toward an SNe Ia. Many different observations show that the reddening law to $\mathrm{SN} 2014 \mathrm{~J}$ has a low value of $R_{V} \approx 1.4-1.6$, and $E(B-V) \approx 0.8-1.3 \mathrm{mag}$ and $A_{V} \sim 1.8-2.0 \mathrm{mag}$ (Amanullah et al. 2014, Foley et al. 2014, Gao et al. 2015). More specifically, Amanullah et al. (2014) determined the reddening curve $E(\lambda-V)$ for SN 2014J over a wide wavelength range, and derived $R_{V} \approx 1.4 \pm 0.1$ by fitting the observationally determined $E(\lambda-V)$ with the Galactic-type law as parameterized by Cardelli et al. (1989; CCM) or Fitzpatrick (1999; FTZ). However, the CCM- and FTZ-parameterizations have been derived for the Galactic sightlines with $2<R_{V}<5$, and may not be valid for external galaxies (Gao et al. 2015).

Using the silicate-graphite dust models, we derive $R_{V}$ and $A_{\lambda}$ toward SN 2014J by fitting the reddening curve obtained by Amanullah et al. (2014). Our model consists of graphite and amorphous silicate, with an exponentially cutoff power-law size distribution (see Gao et al. 2015). Our best-fit models lead to $A_{V} \approx 1.9 \mathrm{mag}, E(B-V) \approx 1.1 \mathrm{mag}$, and $R_{V} \approx 1.7$ to 1.8 . The reddening and $A_{V}$ are generally consistent with those reported earlier, while the model $R_{V}$ values are somewhat larger than most of the earlier results. The model extinction laws for SN 2014J all exhibit a rapid far ultraviolet rise which is even much steeper than that of the SMC bar, and differ substantially from that calculated from the CCM formula (see Figures 2, 3 in Gao et al. 2015). It suggests that one can not directly apply the CCM-formula (or some other parameterizations) to derive $A_{\lambda}$ toward SN 2014J. In Table 1, we present the modeled $A_{\lambda}$ toward SN 2014J derived from the silicate-graphite grain model in Gao et al. (2015) with an elemental depletion of $[\mathrm{Si} / \mathrm{H}]_{\mathrm{dust}}=35.5 \mathrm{ppm}$ and $[\mathrm{C} / \mathrm{H}]_{\mathrm{dust}}=41.6 \mathrm{ppm}$ (see Gao et al. 2015). The model assumes a complete depletion of $\mathrm{Si}, \mathrm{Mg}$, and $\mathrm{Fe}$ and $50 \%$ depletion of $\mathrm{C}$. This is similar to the depletion pattern of the Galaxy.

\section{Acknowledgements}

This work is supported by NSFC 11173007, 11373015, 11533002, 973 Program 2014- CB845702, NSF AST-1109039, NNX13AE63G, and the Fundamental Research Funds for the Central Universities (2015KJJCA03).

\section{References}

Amanullah, R., Goobar, A., Johansson, J., et al. 2014, ApJ (Letters), 788, L21

Cardelli, J. A., Clayton, G. C., \& Mathis, J. S. 1989, ApJ, 345, 245

Fitzpatrick, E. L. 1999, PASP, 111, 63

Foley, R. J., Fox, O. D., McCully, C., et al. 2014, MNRAS, 443, 2887 
Table 1. Model Extinction toward SN 2014J ${ }^{a}$

\begin{tabular}{|c|c|c|c|}
\hline Band & $\begin{array}{c}\lambda \\
(\mu \mathrm{m})\end{array}$ & $\begin{array}{c}A_{\lambda} \\
(\mathrm{mag})\end{array}$ & $\begin{array}{c}\mathrm{C}_{\text {ext }} / \mathrm{H} \\
\left(\mathrm{cm}^{2} / \mathrm{H}\right)\end{array}$ \\
\hline Ly edge & 0.091 & 39.87 & $2.06 \times 10^{-21}$ \\
\hline $\operatorname{Ly} \alpha$ & 0.122 & 29.40 & $1.52 \times 10^{-21}$ \\
\hline UVW2/UVOT & 0.203 & 13.87 & $7.16 \times 10^{-22}$ \\
\hline UVM2/UVOT & 0.223 & 12.53 & $6.47 \times 10^{-22}$ \\
\hline UVW1/UVOT & 0.259 & 8.64 & $4.46 \times 10^{-22}$ \\
\hline F225W/HST & 0.287 & 6.98 & $3.60 \times 10^{-22}$ \\
\hline F275W/HST & 0.290 & 6.84 & $3.53 \times 10^{-22}$ \\
\hline F218W/HST & 0.311 & 5.97 & $3.08 \times 10^{-22}$ \\
\hline F336W/HST & 0.340 & 5.03 & $2.59 \times 10^{-22}$ \\
\hline$u / \operatorname{SDSS}$ & 0.355 & 4.63 & $2.39 \times 10^{-22}$ \\
\hline $\mathrm{U}$ & 0.365 & 4.39 & $2.27 \times 10^{-22}$ \\
\hline F438W/HST & 0.433 & 3.15 & $1.63 \times 10^{-22}$ \\
\hline $\mathrm{B}$ & 0.440 & 3.06 & $1.58 \times 10^{-22}$ \\
\hline F467M/HST & 0.468 & 2.70 & $1.39 \times 10^{-22}$ \\
\hline$g / \mathrm{SDSS}$ & 0.469 & 2.69 & $1.39 \times 10^{-22}$ \\
\hline $\mathrm{V}$ & 0.550 & 1.94 & $1.00 \times 10^{-22}$ \\
\hline F555W/HST & 0.550 & 1.94 & $1.00 \times 10^{-22}$ \\
\hline$r / \mathrm{SDSS}$ & 0.617 & 1.54 & $7.93 \times 10^{-23}$ \\
\hline F631N/HST & 0.630 & 1.48 & $7.62 \times 10^{-23}$ \\
\hline $\mathrm{R}$ & 0.700 & 1.19 & $6.13 \times 10^{-23}$ \\
\hline$i / \mathrm{SDSS}$ & 0.748 & 1.04 & $5.35 \times 10^{-23}$ \\
\hline F814W/HST & 0.792 & 0.92 & $4.75 \times 10^{-23}$ \\
\hline F845M/HST & 0.863 & 0.77 & $3.99 \times 10^{-23}$ \\
\hline$z / \mathrm{SDSS}$ & 0.893 & 0.72 & $3.72 \times 10^{-23}$ \\
\hline$I^{\prime}$ & 0.900 & 0.71 & $3.66 \times 10^{-23}$ \\
\hline $\mathrm{J} / 2 \mathrm{MASS}$ & 1.235 & 0.38 & $1.94 \times 10^{-23}$ \\
\hline $\mathrm{H} / 2 \mathrm{MASS}$ & 1.662 & 0.22 & $1.14 \times 10^{-23}$ \\
\hline $\mathrm{Ks} / 2 \mathrm{MASS}$ & 2.159 & 0.15 & $7.53 \times 10^{-24}$ \\
\hline W1/WISE & 3.353 & 0.08 & $4.32 \times 10^{-24}$ \\
\hline $\mathrm{L}$ & 3.450 & 0.08 & $4.18 \times 10^{-24}$ \\
\hline [3.6]/IRAC & 3.545 & 0.08 & $4.06 \times 10^{-24}$ \\
\hline [4.5]/IRAC & 4.442 & 0.06 & $3.18 \times 10^{-24}$ \\
\hline W2/WISE & 4.603 & 0.06 & $3.07 \times 10^{-24}$ \\
\hline M & 4.800 & 0.06 & $2.96 \times 10^{-24}$ \\
\hline [5.8]/IRAC & 5.675 & 0.05 & $2.78 \times 10^{-24}$ \\
\hline [8.0]/IRAC & 7.760 & 0.10 & $5.20 \times 10^{-24}$ \\
\hline $\mathrm{N}$ & 10.600 & 0.40 & $2.06 \times 10^{-23}$ \\
\hline W3/WISE & 11.561 & 0.27 & $1.40 \times 10^{-23}$ \\
\hline $\mathrm{Q}$ & 21.000 & 0.16 & $8.10 \times 10^{-24}$ \\
\hline W4/WISE & 22.088 & 0.14 & $7.21 \times 10^{-24}$ \\
\hline
\end{tabular}

Gao, J., Jiang, B. W., Li, A., Li, J., \& Wang, X. 2015, ApJ (Letters), 807, L26 